University of Montana

ScholarWorks at University of Montana

1995

\title{
Finiteness of the Fixed Point Set for the Simple Genetic Algorithm
}

Alden H. Wright

University of Montana - Missoula, alden.wright@umontana.edu

Michael D. Vose

Follow this and additional works at: https://scholarworks.umt.edu/cs_pubs

Part of the Computer Sciences Commons

Let us know how access to this document benefits you.

\section{Recommended Citation}

Wright, Alden H. and Vose, Michael D., "Finiteness of the Fixed Point Set for the Simple Genetic Algorithm" (1995). Computer Science Faculty Publications. 8.

https://scholarworks.umt.edu/cs_pubs/8

This Article is brought to you for free and open access by the Computer Science at ScholarWorks at University of Montana. It has been accepted for inclusion in Computer Science Faculty Publications by an authorized administrator of ScholarWorks at University of Montana. For more information, please contact scholarworks@mso.umt.edu. 


\title{
Finiteness of the Fixed Point Set for the Simple Genetic Algorithm
}

\author{
Alden H. Wright ${ }^{1}$ \\ Department of Computer Science \\ The University of Montana \\ Missoula, M'T 59812-1008 \\ wright@cs.umt.edu
}

\author{
Michael D. Vose \\ Department of Computer Science \\ The University of Tennessee \\ Knoxville, 'TN 37996-1301 \\ vose@cs.utk.edu
}

\begin{abstract}
The infinite population simple genetic algorithm is a discrete dynamical system model of a genetic algorithm. It is conjectured that trajectories in the model always converge to fixed points. This paper shows that an arbitrarily small perturbation of the fitness will result in a model with a finite number of fixed points. Moreover, every sufficiently small perturbation of fitness preserves the finiteness of the fixed point set. These results allow proofs and constructions that require finiteness of the fixed point set. For example, applying the stable manifold theorem to a fixed point requires the hyperbolicity of the differential of the transition map of the genetic algorithm, which requires (among other Keywords things) that the fixed point be isolated.

Genetic algorithms, fixed point, transversality, infinite population model, dynamical system.
\end{abstract}

\section{Introduction}

Vose (1990) introduced a rigorous dynamical system model for the binary-representation genetic algorithm with proportional selection, with the simplifying assumption of an infinite population size. This model has been further extended in Vose and Liepins (1991), Vose and Wright (1995), and Vose (in press). If the string length is $\ell$, the model is defined in terms of a differentiable mapping $\mathcal{G}$ from $R^{n}$ into itself, where $n=2^{\ell}$. The mapping $\mathcal{G}$ describes the way a population changes from one generation to the next. The fixed points of $\mathcal{G}$ are of primary importance, because it is conjectured that the iterates of $\mathcal{G}$ always converge to a fixed point. (According to the conjecture, the dynamical system does not show periodic or chaotic behavior. Such behavior has never been observed in practice.) The conjecture is known to be true when the fitness function has low epistasis) (Vose \& Wright, 1995).

An exact Markov chain model for the finite population genetic algorithm was introduced by Nix and Vose (1992) and extended by Vose (1996). This work makes precise the idea that for large population size and positive mutation, a finite population genetic algorithm will spend most of its time near fixed points of the corresponding infinite population genetic algorithm.

We can easily construct examples in which $\mathcal{G}$ has an infinite number of fixed points. For example, this happens when mutation is zero and all strings have the same fitness (Booker, 1993). It is shown in Vose (1990) and Vose (1996) and in Section 5 of this paper, that the fixed

1 This work was done while the first author was visiting the computer science department of the University of Tennessee. 
points of $\mathcal{G}$ are the solutions of a system of $n$ quadratic equations in $n$ unknowns. While such a system of equations may have spaces of solutions, transversality techniques of differential topology can be used to show that a small perturbation of the coefficients of the equations will make the solution set finite. (See, for example, Wright (1985).)

This paper shows that the set of fitness functions such that there are finitely many solutions to $\mathcal{G}(x)=x$ is dense and open. In other words, it is a generic property of fitness that $\mathcal{G}$ has finitely many fixed points; without specific knowledge to the contrary, it is a reasonable assumption because it is the typical case from both a measure-theoretic and a topological perspective. In particular, consequences obtained from the assumption will be the rule rather than the exception.

The importance of finitely many fixed points to the theory of the simple genetic algorithm is mainly that it allows constructions and proof techniques that require finiteness in order to be carried out. For example, applications of the stable manifold theorem (see Vose \& Wright (1995), for instance) rely on the hyperbolicity of the differential of $\mathcal{G}$ at fixed points, which in turn is related to the finiteness of the fixed point set. Moreover, the degree to which the qualitative behavior of a finite population genetic algorithm accords with the dynamical system corresponding to $\mathcal{G}$ is also related to the finiteness of the fixed point set. (This is touched upon in the conclusion of this paper.) The relationship between the finite and infinite population genetic algorithms is further explained in Vose (in press).

\section{The Simple Genetic Algorithm}

The simple genetic algorithm is a standard genetic algorithm over fixed-length binary strings that uses proportional selection and various types of crossover and mutation.

We consider a generalization of the infinite population model of the simple genetic algorithm, introduced in Vose (1990), which is the theoretical framework used in Vose (in press). The domain $\Omega$ is the set of length- $\ell$ binary strings. Let $n=2^{\ell}$ and note that elements of $\Omega$ correspond to integers in the range $[0, n)$. They can thereby be thought of interchangeably as integers or as bit strings that are regarded as column vectors with entries from $\{0,1\}$. Because of frequent use, it is convenient to let 1 denote the vector $n-1$ (which is the vector of all $1 \mathrm{~s}$ ). Let $e_{k}$ denote the $k$ th column of the $n \times n$ identity matrix.

Let $\oplus$ denote the bitwise exclusive-or operation, and let $\otimes$ denote the bitwise and operation, on $\Omega$. For $x \in \Omega$, the ones complement of $x$ is denoted by $\bar{x}$. Note that $\bar{x}=\mathbf{1} \oplus x$.

If expr is an expression that is either true or false, then

$$
[\operatorname{expr}]= \begin{cases}1 & \text { if expr is true } \\ 0 & \text { otherwise }\end{cases}
$$

Let $\delta_{i j}=[i=j]$. The $n \times n$ permutation matrix whose $i, j$ th entry is $\delta_{i \oplus k, j}$ is denoted by $\sigma_{k}$. Note that $\left(\sigma_{k} x\right)_{i}=x_{i \oplus k}$.

A population is a real-valued probability vector $x$ indexed over $\Omega$; the probability (or fraction) of string $i$ in population $x$ is $x_{i}$. The set of all populations is the unit simplex $\Lambda=\left\{x \in\left(R^{\geq 0}\right)^{n}: 1^{T} x=1\right\}$, where $R^{\geq 0}$ denotes the nonnegative reals. The $e_{k}$ are vertices of $\Lambda$ and correspond to populations consisting entirely of one string type (namely $k$ ).

A $n \times n$ mixing matrix $M$ encodes mutation and crossover. $M$ is defined so that $M_{i, j}$ is the probability of obtaining the 0 string by applying mutation and crossover to the parent strings $i$ and $j$. Thus, $x^{T} M x$ is the probability (or fraction) of the 0 string as the result of applying crossover and mutation to population $x$. The formula for the $M$ matrix is given in Vose (in press) and is repeated here for completeness. 
We first give the formula for crossover only (no mutation). Considering $k \in \Omega$ as a crossover mask used with parents $i, j \in \Omega$, the children are $(i \otimes k) \oplus(j \otimes \bar{k})$ and $(j \otimes k) \oplus(i \otimes \bar{k})$. We assume one child is kept (with equal probability). If $\chi_{k}$ denotes the probability that mask $k$ is used, $M$ is given by

$$
M_{i, j}=\sum_{k \in \Omega} \frac{\chi_{k}+\chi_{\bar{k}}}{2}[(i \otimes k) \oplus(j \otimes \bar{k})=0]
$$

We now incorporate mutation. Let $\mu_{i}, i \in \Omega$, be the probability that a string $x$ is mutated into the string $x \rightarrow i$. The standard assumption is that mutation is described by ate $r \in[0,0.5)$. In this case, the $\mu_{i}$ are given by the formula

$$
\mu_{i}=r^{1^{T} i}(1-r)^{\ell-1^{T_{i}}}
$$

Under general crossover and mutation, referred to as mixing, $M$ is given by

$$
M_{i, j}=\sum_{u, v, k \in \Omega} \mu_{u} \mu_{v} \frac{\chi_{k}+\chi_{\bar{k}}}{2}[((i \oplus u) \oplus k) \oplus((j \oplus v) \otimes \bar{k})=0]
$$

Note that $M$ is symmetric and nonnegative. Mutation is said to be positive when $\mu_{i}>0$ for all $i \in \Omega$ (which is the case for a positive mutation rate).

The recombination function $\mathcal{M}: \Lambda \longrightarrow \Lambda$ has component functions defined by

$$
\mathcal{M}_{k}(x)=e_{k}^{T} \mathcal{M}(x)=\left(\sigma_{k} x\right)^{T} M \sigma_{k} x=\sum_{i, j \in \Omega} x_{i} x_{j} M_{i \oplus k_{i} \oplus k}
$$

Then $\mathcal{M}_{k}(x)$ is the probability of string $k$ in the population that is obtained by applying mutation and crossover to population $x$.

The following lemma will be useful later on (in Section 5). In particular, it shows that $\Lambda$ is invariant under $\mathcal{M}$.

LEMMA 2.1: $1^{T} \mathcal{M}(x)=\left(1^{T} x\right)^{2}$

PROOF: First note that $M_{i \oplus k, j \oplus k}$ is the probability of obtaining $k$ by mixing $i$ and $j$. Thus, for all $i, j \in \Omega$,

$$
\sum_{k} M_{i \oplus k j \ominus k}=1
$$

Therefore,

$$
\mathbf{1}^{T} \mathcal{M}(x)=\sum_{k} \sum_{i j} x_{i} x_{j} M_{i \circlearrowleft k_{j} \rho k}=\sum_{i} x_{i} \sum_{j} x_{j} \sum_{k} M_{i \circlearrowleft k_{j} \circlearrowleft k}=\left(\mathbf{1}^{T} x\right)^{2}
$$

Note that $\mathcal{M}$ is bomogeneous of degree 2; that is, $\mathcal{M}(\alpha x)=\alpha^{2} \mathcal{M}(x)$ for any scalar $\alpha$.

Assuming a fitness function $f: \Omega \longrightarrow R^{+}$, proportional selection is the mapping $\mathcal{F}$ : $\Lambda \longrightarrow \Lambda$ defined by $\mathcal{F}(x)=F x / 1^{T} F x$, where $F$ is the $n \times n$ diagonal matrix $F_{i, j}=\delta_{i, j} f(i)$. The fitness function is regarded as a vector through the correspondence $f_{i}=f(i)$.

The transition from one generation to the next of the infinite population simple genetic algorithm is given by the mapping

$$
\mathcal{G}=\mathcal{M} \circ \mathcal{F}: \Lambda \longrightarrow \Lambda
$$

To emphasize the dependence on the fitness function, $\mathcal{G}$ may be written as $\mathcal{G}_{f}$. 


\section{Nonsingularity of Matrices}

In this section, we show how square matrices can be made nonsingular by making arbitrarily small adjustments to their diagonals. These results will later be applied to submatrices of differentials.

LEMMA 3.1: Let $M$ be the partitioned matrix

$$
M=\left(\begin{array}{c|c}
A & B \\
\hline 0 & C
\end{array}\right)
$$

where $A$ and $C$ are square. Then $\operatorname{det}(M)=\operatorname{det}(A) \cdot \operatorname{det}(C)$.

Proof: Induct on the dimension of $A$. If $A$ is $1 \times 1$, the lemma follows directly from an expansion of the determinant by cofactors using the first column of $M$. The inductive step follows, using the same technique, by observing that the inductive hypothesis allows the determinant of $C$ to be factored out of the resulting sum. The remaining factor is the determinant of $A$.

For an $n$-vector $a$, let $\operatorname{diag}(a)$ denote the $n \times n$ diagonal matrix with $i$ th entry $f_{i}$.

THEOREM 3.2: Let $M$ be an $n \times n$ matrix and let $a$ be an $n$-vector. The set

$$
N=\left\{a \in R^{n}: M-\operatorname{diag}(a) \text { is nonsingular }\right\}
$$

is open and dense in $R^{n}$.

Proof: Since $\operatorname{det}(M-\operatorname{diag}(a))$ is a continuous function of $a, N$ is open. The proof is completed by showing that if $a \notin N$, an arbitrarily small perturbation of $a$ is in $N$. The existence of this perturbation is proved by induction on $n$.

The case $n=1$ is trivial. For the inductive step, expand $\operatorname{det}(M-\operatorname{diag}(a))$ by cofactors using the first column. The first term in the resulting sum has the form $\left(M_{0,0}-a_{0}\right) \operatorname{det}(C)$. By the induction hypothesis, a small perturbation of $\left\{a_{1}, a_{2}, \ldots, a_{n-1}\right\}$ makes $\operatorname{det}(C)$ nonzero. Hence this first term is a nonzero polynomial in $a_{0}$. Since no other terms in the expansion involve $a_{0}$, the determinant is a polynomial in $a_{0}$ that can be perturbed away from zero.

\section{Transversality}

This section provides necessary background in differential topology. The treatment here follows Chow, Mallet-Paret, and Yorke (1978). Other references for this material include Milnor (1965), Abraham and Robbin (1967), and Palis and de Melo (1982).

The transversality theorem of differential topology implies that when two manifolds (surfaces) of dimension $k$ and $l$ meet in an $n$-dimensional space, then, after a small adjustment, their intersection will be a manifold of dimension $(k+l-n)$. If $k+l<n$, then one expects the intersection to be empty. Thus, two surfaces in 3 -space would intersect in a curve, and a surface and a curve in 3-space would intersect in a discrete set of points.

For our purposes, we are concerned with the intersection of the image of an $n$-dimensional space and a point in $n$-dimensional space. A point is a 0 -dimensional space, so we would expect a 0 -dimensional intersection. In other words, after a possible small adjustment, the intersection would be discrete. If $g: R^{n} \longrightarrow R^{n}$ is differentiable and $y \in R^{n}$ is a given point, a small adjustment of $g$ will make the inverse image of $y$ under $g$ a discrete set of points. 
A map $g: U \subseteq R^{m} \longrightarrow R^{n}$ is $C^{r}$ if the $r$ th partial derivatives of the component functions of $g$ exist and are continuous. ${ }^{2}$ A point $x \in U$ is regular if the differential $d g_{x}$ is surjective; otherwise $x$ is critical. A point $y \in R^{n}$ is a regular value if every $x \in g^{-1}(y)$ is a regular point; otherwise, $y$ is a critical value.

THEOREM 4.1: Suppose $g: U \subseteq R^{n} \longrightarrow R^{n}$ is $C^{1}$ and $x \in U$ is a regular point of $g$. Then there is a neigbborbood $V$ of $x$ such that $V \cap g^{-1}(y)=x$, where $y=g(x)$.

ProOF: By surjectivity, the differential $d g_{x}: R^{n} \longrightarrow R^{n}$ is nonsingular. Hence the inverse function theorem implies that $g$ is a local diffeomorphism.

The next theorem, which is taken from Chow, Mallet-Paret, and Yorke (1978), can be viewed either as a generalization of Sard's theorem or as a special case of a transversality theorem.

THEOREM 4.2: (Parameterized Sard's Theorem) Let $V \subseteq R^{q}$ and $U \subseteq R^{m}$ be open, and suppose $H: V \times U \longrightarrow R^{n}$ is $C^{r}$ where $r>\max (0, m-n)$. If $0 \in R^{n}$ is a regular value of $H, 0$ is $a$ regular value of $H_{a}: U \longrightarrow R^{n}$ for almost all $a \in V$, where $H_{a}(\cdot)=H(a, \cdot)$.

THEOREM 4.3: Let $V \subseteq R^{q}$ be open, let $K \subseteq R^{m}$ be compact, and let $H: V \times K \longrightarrow R^{m}$ be $C^{1}$. Then the set $\left\{b \in V: 0\right.$ is a regular value of $\left.H_{b}\right\}$ is open in $V$, where $H_{b}(\cdot)=H(b, \cdot)$.

ProOf: Choose $a \in V$ so that 0 is a regular value of $H_{a}$. For each $x \in K$ such that $H_{a}(x)=0$, let $U_{x} \subseteq K$ be an open neighborhood of $x$ such that $\operatorname{det}\left(d\left(H_{a}\right)_{y}\right) \neq 0$ for all $y \in \bar{U}_{x}$. Let $W_{x} \subset V$ be a neighborhood of $a$ such that $\operatorname{det}\left(d\left(H_{b}\right)_{y}\right) \neq 0$ for all $b \in W_{x}$ and all $y \in \bar{U}_{x}$. Let $U_{x_{1}}, U_{x_{2}}, \ldots, U_{x_{r}}$ be a finite open cover of $H_{a}^{-1}(0) \subseteq K$. Let $U=U_{x_{1}} \cup U_{x_{2}} \cup \cdots \cup U_{x_{r}}$, and let $W=W_{x_{1}} \cap W_{x_{2}} \cap \cdots \cap W_{x_{r}}$. Then $\operatorname{det}\left(d\left(H_{b}\right)_{y}\right) \neq 0$ for all $b \in W$ and $y \in U$. Since $0 \notin H_{a}(K \backslash U)$, we can choose a neighborhood $W^{\prime} \subseteq W$ of $a$ such that $0 \notin H_{b}(K \backslash U)$ for all $b \in W^{\prime}$. Thus, for every $b \in W^{\prime}, 0$ is a regular value of $H_{b}$.

\section{Equations for Fixed Points}

We show how fixed points of $\mathcal{G}$ can be found by solving the system of quadratic equations

$$
\mathcal{M}(x)=F^{-1} x
$$

In Section 7, we will apply transversality to $\mathcal{H}: R^{n} \longrightarrow R^{n}$ defined by $\mathcal{H}(x)=\mathcal{M}(x)-F^{-1} x$ to show that after a small adjustment of $\mathcal{H}, \mathcal{H}^{-1}(0)$ is a discrete set of points. Note that $\mathcal{H}^{-1}(0)$ is the set of fixed points of $\mathcal{G}$.

The material of this section is adapted from Vose (1990) and Vose (1996).

Proposition 5.1: A nonzero $x \in\left(R^{\geq 0}\right)^{n}$ satisfies $\mathcal{M}(x)=F^{-1} x$ if and only if $\left(1^{T} x\right)^{-2} F^{-1} x$ is a fixed point of $\mathcal{G}$.

PROOF: A simple computation shows that $y=\left(\mathbf{1}^{T} x\right)^{-2} F^{-1} x$ is equivalent to $x=\left(1^{T} F y\right)^{-2} F y$.

Suppose $\mathcal{G}(y)=y$ and let $x=\left(\mathbf{1}^{T} F y\right)^{-2} F y$. Then

$$
\mathcal{M}(x)=\mathcal{M}\left(\frac{F y}{\left(\mathbf{1}^{T} F y\right)^{2}}\right)
$$

2 This may require that $g$ be defined on some open set strictly containing $U$. 
Alden H. Wright and Michael D. Vose

$$
\begin{aligned}
& =\frac{1}{\left(\mathbf{1}^{T} F y\right)^{2}} \mathcal{M}\left(\frac{F y}{\mathbf{1}^{T} F y}\right) \\
& =\frac{y}{\left(\mathbf{1}^{T} F y\right)^{2}} \\
& =F^{-1} \frac{F y}{\left(\mathbf{1}^{T} F y\right)^{2}} \\
& =F^{-1} x
\end{aligned}
$$

Conversely, suppose that nonzero $x \in\left(R^{\geq 0}\right)^{n}$ satisfies $\mathcal{M}(x)=F^{-1} x$ and let $y=\left(1^{T} x\right)^{-2} F^{-1} x$. These same equalities (the second and third lines in particular) show that $\mathcal{G}(y)=y$.

PROPOSITION 5.2: If $x \in\left(R^{\geq 0}\right)^{n}$ is a nonzero solution of $\mathcal{M}(x)=F^{-1} x$,

$$
\min f_{i}^{-1} \leq \mathbf{1}^{T} x \leq \max f_{i}^{-1}
$$

PROOF: First, note that

$$
\left(\mathbf{1}^{T} x\right) \min f_{i}^{-1} \leq \sum x_{i} f_{i}^{-1} \leq\left(\mathbf{1}^{T} x\right) \max f_{i}^{-1}
$$

Combining Lemma 2.1 with $\mathcal{M}(x)=F^{-1} x$ gives

$$
\mathbf{1}^{T} F^{-1} x=\left(1^{T} x\right)^{2}
$$

Putting these two facts together yields $\min f_{i}^{-1} \leq\left(1^{T} x\right) \leq \max f_{i}^{-1}$.

\section{Linear Subspaces and Cones}

This section brings together some definitions and technical results concerning when the mixing map $\mathcal{M}$ preserves the positive orthant of a coordinate subspace of $R^{n}$. These will be useful in establishing the main results (given in Section 7).

For $\Phi \subseteq \Omega$, define $\mathcal{L}(\Phi)$ to be the linear span of the set of basis vectors $\left\{e_{k}: k \in \Phi\right\}$. Clearly, the dimension of $\mathcal{L}(\Phi)$ is the cardinality of $\Phi$. Define $\mathcal{C}(\Phi)$ to be the cone generated by $\left\{e_{k}: k \in \Phi\right\}$,

$$
\mathcal{C}(\Phi)=\left\{\sum_{k \in \Phi} \alpha_{k} e_{k}: \alpha_{k} \geq 0\right\}
$$

and let $\mathcal{C}^{\circ}(\Phi)$ be its interior,

$$
\mathcal{C}^{\circ}(\Phi)=\left\{\sum_{k \in \Phi} \alpha_{k} e_{k}: \alpha_{k}>0\right\}
$$

LEMMA 6.1: Let $\Phi \subseteq \Omega$. If $\Phi$ is nonempty, exactly one of the following bolds:

1. $\mathcal{M}\left(\mathcal{C}^{\circ}(\Phi)\right) \subseteq \mathcal{C}^{\circ}(\Phi)$

2. $\mathcal{M}\left(\mathcal{C}^{\circ}(\Phi)\right) \cap \mathcal{C}^{\circ}(\Phi)=\emptyset$ 
ProOf: Suppose $x \in \mathcal{C}^{\circ}(\Phi)$ but $\mathcal{M}(x) \notin \mathcal{C}^{\circ}(\Phi)$. We will show $\mathcal{M}\left(\mathcal{C}^{\circ}(\Phi)\right) \cap \mathcal{C}^{\circ}(\Phi)=\emptyset$. There are two possibilities:

$$
\begin{aligned}
& \text { 1. } \mathcal{M}(x) \notin \mathcal{C}(\Phi) \\
& \text { 2. } \mathcal{M}(x) \in \mathcal{C}(\Phi) \backslash \mathcal{C}^{\circ}(\Phi)
\end{aligned}
$$

In the first case, there exists $k \notin \Phi$ such that $e_{k}^{T} \mathcal{M}(x)>0$, since $\mathcal{M}$ maps the nonnegative orthant $\mathcal{C}(\Omega)$ into itself. Recall that

$$
e_{k}^{T} \mathcal{M}(x)=\sum_{i, j \in \Phi} x_{i} x_{j} M_{i \oplus k, j k}
$$

Thus, there must exist $i, j \in \Phi$ for which $M_{i \oplus k_{2}, j k}>0$. It follows that $e_{k}^{T} \mathcal{M}(y)>0$ for any $y \in \mathcal{C}^{\circ}(\Phi)$; this implies $\mathcal{M}\left(\mathcal{C}^{\circ}(\Phi)\right) \cap \mathcal{C}^{\circ}(\Phi)=\emptyset$.

In the second case, there exists $k \in \Phi$ such that $e_{k}^{T} \mathcal{M}(x)=0$. Thus, $M_{i \oplus k, j(j) k}=0$ for every $i, j \in \Phi$. It follows that $e_{k}^{T} \mathcal{M}(y)=0$ for all $y \in \mathcal{C}^{\circ}(\Phi)$; this implies $\mathcal{M}\left(\mathcal{C}^{\circ}(\Phi)\right) \cap \mathcal{C}^{\circ}(\Phi)=\emptyset$.

REMARK: The second case in the preceding proof can happen only if $M_{0,0}=0$. But for any reasonable choice of crossover and mutation, $M_{0,0} \neq 0$. The first case in the preceding proof establishes the stronger conclusion that either $\mathcal{M}\left(\mathcal{C}^{\circ}(\Phi)\right) \subseteq \mathcal{C}^{\circ}(\Phi)$ or $\mathcal{M}\left(\mathcal{C}^{\circ}(\Phi)\right) \cap \mathcal{L}(\Phi)=\emptyset$.

LEMMA 6.2: If $\mathcal{M}\left(\mathcal{C}^{\circ}(\Phi)\right) \subseteq \mathcal{L}(\Phi), \mathcal{M}(\mathcal{L}(\Phi)) \subseteq \mathcal{L}(\Phi)$

PROOF: For each $k \notin \Phi, e_{k}^{T} \mathcal{M}(x)$ is polynomial. Since $\mathcal{C}^{\circ}(\Phi)$ is an open subset of $\mathcal{L}(\Phi)$ and $e_{k}^{T} \mathcal{M}(x)$ is 0 on this open subset, it must be 0 on all of $\mathcal{L}(\Phi)$.

RFMARK: When mutation is positive, $M>0$ and hence $\mathcal{M}\left(\mathcal{C}^{\circ}(\Phi)\right) \subseteq \mathcal{C}^{\circ}(\Omega)$ is easily verified. If, in addition, $\Phi$ is a nonempty proper subset of $\Omega$, it is easy to show that $\mathcal{M}\left(\mathcal{C}^{\circ}(\Phi)\right) \cap \mathcal{L}(\Phi)=\emptyset$ and $\mathcal{M}(\mathcal{C}(\Phi)) \cap \mathcal{L}(\Phi)=\{0\}$.

\section{Fixed Points in the Interior of the Simplex}

This section presents the main result of this paper in Corollary 7.3 .

It is convenient to regard $a_{i}=1 / f_{i}$ as variables. Define $\mathcal{H}(a, x)=\mathcal{H}_{a}(x)=\mathcal{M}(x)-\operatorname{diag}(a) x$. By Proposition 5.1, $x$ is a fixed point of $\mathcal{G}_{f}$ if and only if $\mathcal{H}_{a}(x)=0$. The notation function |set used below indicates that function is to be considered as having domain set.

THEOREM 7.1: There is an arbitrarily small perturbation of the inverse fitness vector a such that 0 is a regular value of $\mathcal{H}_{a}$.

NOTE: Since we have assumed $f>0$, an arbitrarily small perturbation of $a$ is equivalent to an arbitrarily small perturbation of fitness.

The reason for proving Theorem 7.1 is to establish it sequentially for the faces of $\mathcal{C}(\Omega)$ in order of dimension. The main work is proving the next lemma.

LEMMA 7.2: Let $\Phi \subseteq \Omega$, and let $a \in\left(R^{\geq 0}\right)^{n}$ be sucb that 0 is a regular value of $\mathcal{H}_{a} \mid \mathcal{C}(\Phi) \backslash \mathcal{C}^{\circ}(\Phi)$. Then there is an arbitrarily small perturbation of a that makes 0 a regular value of $\mathcal{H}_{a} \mid \mathcal{C}(\Phi)$.

ProOF: If $\mathcal{M}\left(\mathcal{C}^{\circ}(\Phi)\right) \cap \mathcal{C}^{\circ}(\Phi)=\emptyset, \mathcal{C}^{\circ}(\Phi) \cap \mathcal{H}_{a}^{-1}(0)=\emptyset$, so the lemma is true by default. Hence we may assume that $\mathcal{M}\left(\mathcal{C}^{\circ}(\Phi)\right) \subseteq \mathcal{C}^{\circ}(\Phi)$ by Lemma 6.1, and therefore that $\mathcal{H}_{b}(\mathcal{L}(\Phi)) \subseteq \mathcal{L}(\Phi)$ 
for all $b$ (by Lemma 6.2). Define the compact set $K \subseteq R^{n}$ by

$$
K=\left\{x \in \mathcal{C}(\Omega): \frac{1}{2} \min a_{i} \leq 1^{T} x \leq 2 \max a_{i}\right\}
$$

By Proposition 5.2, we may choose a neighborhood of $a \in R^{n}$ such that for any $b$ in this neighborhood, $\mathcal{H}_{b}^{-1}(0)$ lies in $K$. We suppose all subsequent perturbations of $a$ are contained within this neighborhood.

The differential $d \mathcal{H}_{a, x}$ is the $n \times 2 n$ partitioned matrix $\left(-\operatorname{diag}(x) \mid d \mathcal{M}_{x}-\operatorname{diag}(a)\right)$. Let $m=|\Phi|$, denote the inclusion mapping from $\mathcal{L}(\Phi)$ into $R^{n}$ by $i$, and denote the projection from $R^{n}$ onto $\mathcal{L}(\Phi)$ by $\pi$.

Define $\tilde{\mathcal{H}}: \mathcal{L}(\Phi) \times \mathcal{L}(\Phi) \longrightarrow \mathcal{L}(\Phi)$ by $\tilde{\mathcal{H}}(b, y)=\pi(\mathcal{H}(i(b), i(y)))$. It is not hard to confirm that 0 is a regular value of $\widetilde{\mathcal{H}}_{\pi(a)} \mid \mathcal{C}(\Phi) \backslash \mathcal{C}^{\circ}(\Phi)$. The differential $d \widetilde{\mathcal{H}}_{\pi(a), y}$ is the $m \times 2 m$ matrix

$$
\left(-\operatorname{diag}(y) \mid d \widetilde{\mathcal{M}}_{y}-\operatorname{diag}(\pi(a))\right)
$$

where $\widetilde{\mathcal{M}}$ is defined from $\mathcal{M}$ in the same way as $\widetilde{\mathcal{H}}$ is defined from $\mathcal{H}$.

Since $y>0$ for all $y \in \mathcal{C}^{\circ}(\Phi)$, this matrix has rank $m$ for all such $y$. Hence 0 is a regular value of $\widetilde{\mathcal{H}} \mid \mathcal{L}(\Phi) \times \mathcal{C}^{\circ}(\Phi)$. Theorem 4.2 says that an arbitrarily small perturbation of $\pi(a)$ will make 0 a regular value of $\widetilde{\mathcal{H}}_{\pi(a)} \mid \mathcal{C}^{\circ}(\Phi)$. By Theorem 4.3 , this perturbation may be sufficiently small that 0 remains a regular value of $\widetilde{\mathcal{H}}_{\pi(a)} \mid K \cap\left(\mathcal{C}(\Phi) \backslash \mathcal{C}^{\circ}(\Phi)\right)$. It follows that 0 is a regular value of $\widetilde{\mathcal{H}}_{\pi(a)} \mid \mathcal{C}(\Phi)$ for a small perturbation of $a$, where only those coordinates of $a$ indexed by $\Phi$ are perturbed. The next step is to show that under a suitable permutation of the remaining coordinates of $a, 0$ is a regular value of $\mathcal{H}_{a} \mid \mathcal{C}(\Phi)$.

First, note that for the perturbed $a$ (as above), $\mathcal{C}^{\circ}(\Phi) \cap \widetilde{\mathcal{H}}_{\pi(a)}^{-1}(0)$ is finite. Otherwise, $\mathcal{C}(\Phi) \cap \widetilde{\mathcal{H}}_{\pi(a)}^{-1}(0)$ contains an accumulation point (since $K \cap \mathcal{C}(\Phi)$ is compact), which violates Theorem 4.1. It follows that $\mathcal{C}(\Phi) \cap \mathcal{H}_{a}^{-1}(0)$ is also finite, since $\mathcal{H}_{a}\left|\mathcal{C}(\Phi)=\widetilde{\mathcal{H}}_{\pi(a)}\right| \mathcal{C}(\Phi)$.

Let $x \in \mathcal{C}(\Phi) \cap \mathcal{H}_{a}^{-1}(0)$. We now show that the remaining coordinates of $a$ (those not indexed by elements of $\Phi$ ) can be perturbed so that $d\left(\mathcal{H}_{a}\right)_{x}$ is nonsingular-that is, so that $x$ is a regular point of $\mathcal{H}_{a}$.

Reorder the standard basis for $R^{n}$ so that those basic elements corresponding to elements of $\Phi$ come first. Under this ordering, the differential $d\left(\mathcal{H}_{a}\right)_{x}$ is represented by a partitioned matrix of the form

$$
\left(\begin{array}{c|c}
d\left(\tilde{\mathcal{H}}_{\pi(a)}\right)_{\pi(x)} & D \\
\hline 0 & E
\end{array}\right)
$$

The lower left block is zero, since $H_{a}(\mathcal{L}(\Phi)) \subseteq \mathcal{L}(\Phi)$, and we have already perturbed $a$ (as above) so that $d\left(\widetilde{\mathcal{H}}_{\pi(a)}\right)_{\pi(x)}$ is nonsingular. Since $d\left(\mathcal{H}_{a}\right)_{x}$ is also of the form $d \mathcal{M}_{x}-\operatorname{diag}(a)$, Theorem 3.2 applies to the submatrix $E$. Hence there is an arbitrarily small perturbation of the coordinates of $a$ not indexed by elements of $\Phi$, making $E$ nonsingular. Once $E$ is nonsingular, Lemma 3.1 implies that the differential is nonsingular.

This argument can be applied successively to the finitely many points of $\mathcal{C}(\Phi) \cap \mathcal{H}_{a}^{-1}(0)$. By Theorem 4.3, the perturbations, if sufficiently small, will preserve the regularity of previously considered points.

ProOF OF TheOREM 7.1: Consider the cardinality-1 subsets of $\Omega$, which correspond to the vertices $e_{0}, e_{1}, \ldots, e_{n-1}$ of $\Lambda$. For $k=0,1, \ldots, n-1$, we perturb $a$ so that $d\left(\mathcal{H}_{a}\right)_{e_{k}}$ is nonsingular. 
$\left(d\left(\mathcal{H}_{a}\right)_{e_{k}}\right.$ is of the form $d \mathcal{M}_{e_{k}}-\operatorname{diag}(a)$, so we can apply Theorem 3.2). Theorem 4.3 ensures that these perturbations can be chosen to preserve the regularity of previously considered points. Thus, we may suppose $a$ has been perturbed so that each $d\left(\mathcal{H}_{a}\right)_{e_{k}}$ is nonsingular.

Choose an ordering of the subsets of $\Omega$ of cardinality greater than 1 so that subsets are ordered by increasing cardinality. This corresponds to an ordering of the faces of $\mathcal{C}(\Omega)$ so that lower-dimension faces precede higher-dimension faces. Apply Lemma 7.2 to each face in turn. 'The final face is $\mathcal{C}(\Omega)$ itself, so the final application of Lemma 7.2 completes the proof.

REMARK: If mutation is positive, the proof of Theorem 7.1 is very much simplified. As we noted in Section $6, \mathcal{M}$ (and hence $\mathcal{H}$ ) maps $\mathcal{C}(\Omega)$ into $\mathcal{C}^{\circ}(\Omega)$, so Theorems 4.2 and 4.1 can be applied directly to prove the theorem.

COROLLARY 7.3: The set of $\in\left(R^{>0}\right)^{n}$ such that 0 is a regular value of $\mathcal{H}_{f}$ is open and dense. For each such $f, \mathcal{G}_{f}$ bas a finite number of fixed points.

Proof: Openness follows from Theorem 4.3 and denseness follows from Theorem 7.1. The finiteness of the set of fixed points of $\mathcal{G}_{f}$ follows from Theorem 4.1.

\section{Conclusion}

Although it is beyond the scope of this paper to thoroughly show how the finiteness of the fixed point set is related to the degree to which the qualitative behavior of a finite population genetic algorithm accords with the dynamical system corresponding to $\mathcal{G}$, a few brief remarks in that area will be made here.

A finite population GA has population transitions that are essentially perturbations according to a multinormal distribution from

$$
p \mapsto \mathcal{G}(p)
$$

This is illustrated by Figure 1.

The thick arrow represents the transition according to $\mathcal{G}$; the thin arrow represents a possible transition taken by a finite population GA. The distribution of transitions of the

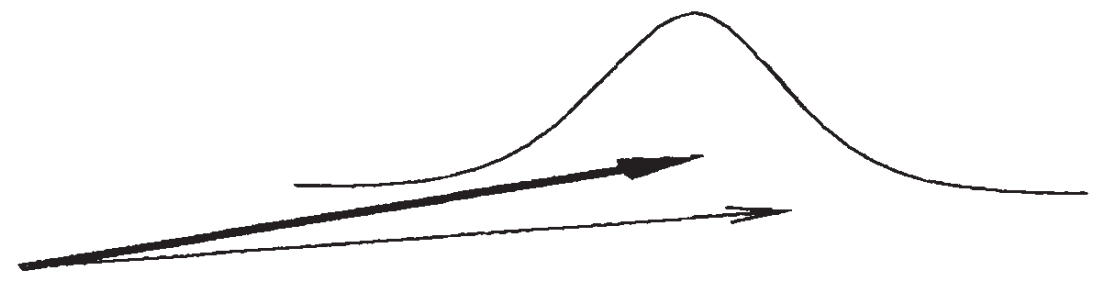

Figure 1. Transitions according to $\mathcal{G}$ and a finite population GA. The thick arrow is the transition according to $\mathcal{G}$; the thin arrow is the transition according to a finite population GA. 


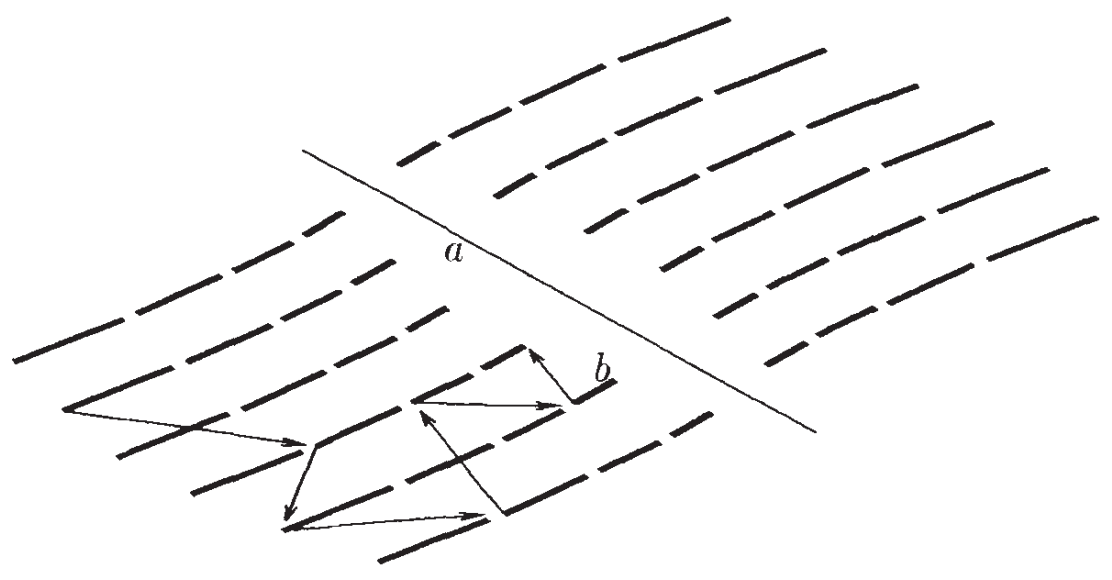

Figure 2. Transitions near a continuum of fixed points. The thick lines represent transitions of $\mathcal{G}$; the thin arrows represent transitions of a finite population GA.

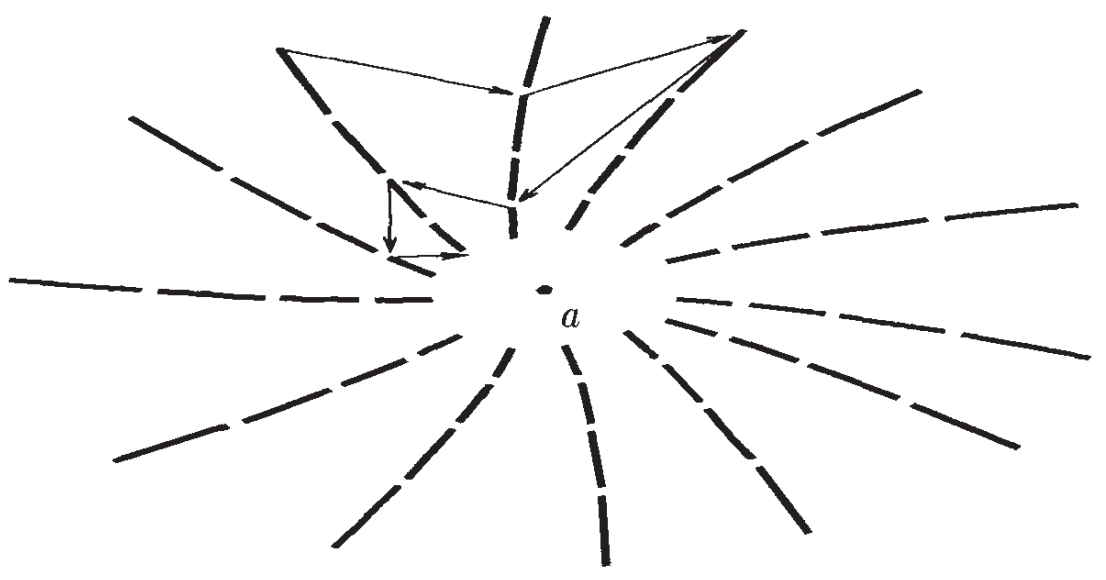

Figure 3. Transitions near an isolated attracting fixed point $a$.

finite population GA is multinomial-centered at the transition by $\mathcal{G}$. The curve schematically indicates the multinormal approximation to this multinomial distribution.

In the case of a continuum of fixed points, these "sampling errors" (deviations from $\mathcal{G}$ ) can accumulate, as in Figure 2, to yield large differences in the evolutionary trajectories.

As in Figure 1, the thick lines represent transitions according to $\mathcal{G}$, and the thin arrows represent those taken by a finite population GA. The thin line in the center represents an "attracting" continuum of fixed points. The transitions of $\mathcal{G}$ are toward this continuum of fixed points. Although evolution toward $a$ is predicted by $\mathcal{G}$, a finite population GA may in fact be tending toward $b$. The underlying dynamical system biases trajectories to move toward the fixed point set, but because no bias is provided in the orthogonal direction, lateral drift (due to stochastic effects) is to be expected.

The analogous situation can be significantly different in the case of finitely many fixed points. A view of possible dynamics in a neighborhood of an attracting fixed point is represented in Figure 3. 
The thick lines represent transitions of $\mathcal{G}$ toward the attracting fixed point $a$. Here, there is no direction in which stochastic drift is not biased toward the fixed point. Evolution toward $a$ is expected in both the infinite and the finite population GAs.

This paper shows that the set of fitness functions such that there are finitely many solutions to $\mathcal{G}(x)=x$ is dense and open. In other words, it is a generic property; without specific knowledge to the contrary, it is a reasonable assumption that $\mathcal{G}$ has finitely many fixed points, since that is the typical case from both a measure-theoretic ${ }^{3}$ and a topological perspective.

\section{Acknowledgments}

The first author was partially supported by a Montana NSF EPSCOR grant and a University of Montana University Research grant. The second author was supported by the National Science Foundation: IRI-8917545 and CDA-9115428.

\section{References}

Abraham, R., \& Robbin, J. (1967). Transversal mappings and flows. New York: W. A. Benjamin.

Booker, L. (1993). Recombination distributions for genetic algorithms. In Foundations of Genetic Algoritbms 2. San Mateo, CA: Morgan Kaufmann.

Chow, S., Mallet-Paret, J., \& Yorke, J. A. (1978). Finding zeros of maps: Homotopy methods that are constructive with probability one. Matbematics of Computation, 31, 887-899.

Milnor, J. W. (1965). Topology from the differentiable viewpoint. Charlottesville, VA: University Press of Virginia.

Nix, A., \& Vose, M. D. (1992). Modeling genetic algorithms with Markov chains. Annals of Matbematics and Artificial Intelligence, 5, 79-88.

Palis, J., \& de Melo, W. (1982). Geometric theory of dynamical systems, an introduction. New York: Springer-Verlag.

Vose, M. D. (1990). Formalizing genetic algorithms. Proc. IEEE Workshop on G.A.s, N.N.s, and S.A. Applied to Problems in Signal and Image Processing, May 1990. Glasgow, U.K.

Vose, M. D. (1996). Models of genetic algorithms. (Submitted to Evolutionary Computation).

Vose, M. D. (in press). The simple genetic algorithm: Foundations and theory. Cambridge, MA: MIT Press.

Vose, M. D., \& Liepins, G. E. (1991). Punctuated equilibria in genetic search. Complex Systems, 5, 31-44.

Vose, M. D., \& Wright, A. H. (1995). Simple genetic algorithms with linear fitness. Evolutionary Computation, 2(4), 347-368.

Wright, A. H. (1985). Finding all solutions to a system of polynomial equations. Mathematics of Computation, 44, 125-133.

3 Assuming absolute continuity with respect to Lebesgue measure. 